\title{
CARACTERIZAÇÃO FLORÍSTICA DA VEGETAÇÃO LENHOSA DE UM FRAGMENTO URBANO DE FLORESTA OMBRÓFILA MISTA ALTO MONTANA, CAMPOS DO JORDÃO, SP
}

\author{
FLORISTIC CHARACTERIZATION OF WOODY \\ VEGETATION OF AN URBAN FRAGMENT OF \\ "FLORESTA OMBRÓFILA MISTA ALTO MONTANA" \\ (TROPICAL MONTANE FOREST), CAMPOS DO \\ JORDÃO, SP
}

\section{Pereira-Silva, E. F. L. ${ }^{1}$, Hardt, E. ${ }^{2}$; Francisco, C. E. da S. ${ }^{3}$}

${ }^{1}$ Programa de Pós-graduação em Biologia Vegetal, Universidade Estadual de Campinas, Caixa Postal 6109, 13083-971 Campinas, SP, Brasil. E-mail: candeia@email.com

${ }^{2}$ Programa de Pós-graduação em Engenharia Civil, Universidade Estadual de Campinas, Caixa Postal 6021, CEP13083-852 Campinas, SP, Brasil.

${ }^{3}$ Programa de Pós-graduação em Agricultura Tropical e Subtropical, Instituto Agronômico de Campinas, Caixa Postal 28, CEP 13001-970 Campinas, SP, Brasil.

\section{RESUMO}

Foram realizados o levantamento florístico e a classificação em grupos de síndromes de dispersão e de sucessão ecológica das espécies lenhosas ocorrentes em um fragmento florestal de 1,14ha, localizado em de Campos do Jordão, SP. O levantamento florístico registrou 54 espécies lenhosas pertencentes a 43 gêneros de 33 famílias ocorrente na borda, no interior e em ambos os hábitats do remanescente florestal. Prevaleceu uma maior proporção de espécies zoocóricas $(31,4 \%)$, ocorrendo espécies anemocóricas $(23,5 \%)$ e autocóricas (3,9\%), indicando uma proporção comum às florestas tropicais. A classificação sucessional indicou o predomínio de espécies secundárias (44\%), seguido pelas pioneiras (18\%), além de diversas espécies sem caracterização $(38 \%)$ devido à carência de informações a respeito de suas estratégias de regeneração. Possivelmente os impactos negativos do processo de fragmentação atuante na região foram, provavelmente, os fatores determinantes para o empobrecimento florístico e a descaracterização de sua fitofisionomia original de Floresta Ombrófila Mista Alto Montana que se encontra em estágio intermediário de regeneração. Apesar do baixo número de espécies registradas na área, esse fragmento 
pode ser de grande importância para a sustentabilidade dos remanescentes vizinhos se comprovado seu papel na conectividade funcional da paisagem. Nesse remanescente florestal, ainda podem ser encontradas espécies arbustivas e arbóreas comuns às formações florestais estacionais e mistas de altitude, que demonstram sua função de manutenção da ameaçada biodiversidade regional.

Palavras-chave: Fragmento florestal. Florística. Espécies lenhosas. Floresta Ombrófila Mista. Alto Montana. Campos do Jordão.

\begin{abstract}
A floristic survey was conducted of woody species occurring in a 1.14ha forest fragment located in Campos do Jordão, SP, which were classified according to dispersion syndrome and ecological succession. Fifty-four woody species pertaining to 43 genera and 33 families were recorded, occurring on the edge, the interior, and in both habitats in the forest fragment. Zoochory was the most frequent species (31.37\%), followed by anemochory $(23.5 \%)$ and autochory $(3.9 \%)$. The successional classification indicated the predominance of secondary species $(44 \%)$ followed by pioneers $(18 \%)$, as well as diverse species without characterization $(38 \%)$ due to the lack of information regarding their regeneration strategies. Negative impacts of the fragmentation process acting in the region were probably determining factors in the floristic impoverishment and damage in the tropical montane forest fragment in intermediate of phase of regeneration. Despite the low number of species recorded, this fragment can be of great importance for the sustainability of the surrounding forest fragments if its role in the functional connectivity of the landscape is proven. In this forest fragment, woody species common to this kind mountain forests formation can still be found, demonstrating its function in the maintenance of threatened regional biodiversity.

Keywords: Forest fragment. Floristic. Woody species. Tropical Montane Forest. Campos do Jordão.
\end{abstract}

\title{
1. INTRODUÇÃO
}

Originalmente, o Estado de São Paulo apresentava mais de $82 \%$ de sua área coberta por florestas contínuas, restando hoje cerca de $7 \%$ dessa cobertura (FUNDAÇÃO SOS MATA ATLÂNTICA, 1998) que é formada por fragmentos florestais de diversos tamanhos, formas, estádios de sucessão e estado de conservação, cada vez mais isolados uns dos outros, geralmente restritos às áreas não produtivas e muito suscetíveis às alterações antrópicas do fogo, do extrativismo e da especulação imobiliária. 
Esses fragmentos respondem de diversas maneiras às perturbações, dependendo do tipo, da idade e da regularidade da modificação, além de dependerem do grau de isolamento da paisagem e do tamanho do fragmento (RODRIGUES, 1995).

Como agente perturbador, o crescimento populacional das cidades exerce uma constante pressão sobre a vegetação urbana com impactos quantitativos, quando diminuem a cobertura vegetal e, qualitativos, quando alteram a composição dos táxons (RUSCZYK, 1986) e tem sua diversidade original substituída por um número muito reduzido de espécies (NORGAARD, 1997). Para a ciência, ainda não está claro o que determina o desaparecimento de uma espécie mais rapidamente do que outra e até que ponto isso pode ser um fenômeno previsível (MORELLATO, 1995), mas não há dúvida de que a fragmentação sempre resulta na diminuição da diversidade ocasionada pela extinção local de espécies (RODRIGUES, 1995).

Mesmo perturbados, os fragmentos em áreas urbanas são muitas vezes sítios de significativo valor florístico que necessitam de proteção. Sua diversidade de espécies vegetais possibilita a instalação de uma fauna diversificada (CESTARO, 1985), importante para o aumento da complexidade estrutural e da resistência às variações ambientais e aos impactos negativos (WERNECK, 1998).

A região da Serra da Mantiqueira é exemplo dessa situação. Detentora de importantes atributos naturais e de elevada diversidade vegetal e animal têm sofrido com as interferências negativas geradas pela facilidade de acesso à região, pela intensificação do turismo e pela demanda de áreas para construções residenciais, que elevam o preço da terra e a especulação imobiliária (SÃO PAULO, 2006). Essas interferências já ocasionaram a fragmentação de duas importantes fisionomias florestais ocorrentes na região, a Floresta Ombrófila Densa Alto Montana e a Floresta Ombrófila Mista Alto Montana, segmentadas atualmente em 954 remanescentes (SÃO PAULO, 2006).

Município de Campos do Jordão abriga 32,3\% dos fragmentos de vegetação natural de Floresta Ombrófila Mista Alto Montana da Serra da Mantiqueira (SÃ̃ PAULO, 2006). A maioria dos fragmentos está em processo de descaracterização, com áreas inferiores a 200 hectares (ha) e compõem a Área de Proteção Ambiental (APA) de Campos do Jordão (SÃO PAULO, 2006). Apenas sete fragmentos $(0,74 \%)$ desse município são maiores que $200 \mathrm{ha}$ e três deles compõem as Unidades de Conservação do Parque Estadual de Campos do Jordão (PECJ), com 8.341ha, do Parque Estadual dos Mananciais de Campos do Jordão, com 502,96ha, e do Parque Ecológico "Erna Schmidt”, com 48ha (SÃO PAULO, 2006).

Tanto as estratégias de conservação quanto a importância desses fragmentos urbanos como reservas naturais de diversidade são temáticas pouco discutidas na esfera dos órgãos competentes. Carecem informações sobre a composição florística e sobre a estrutura desses remanescentes florestais, os quais serviriam como base para estudos sinecológicos e autoecológicos que constituiriam informações essenciais à recomposição e ao restabelecimento desses fragmentos urbanos. 
Considerando essa carência de conhecimento, este trabalho teve como objetivo realizar o levantamento florístico dos estratos arbustivo e arbóreo de um fragmento florestal localizado em uma região de notável expansão urbana no interior do Estado de São Paulo, fornecendo informações sobre seu estado de conservação.

\section{MATERIAL E MÉTODOS}

O fragmento florestal estudado localiza-se no perímetro urbano do Município de Campos do Jordão, o qual se encontra em uma zona de contato entre três regiões florísticas, a Mata de Araucaria-Podocarpus, marcada pela presença de Podocarpus lambertii Klotz. e de Araucaria angustifolia (Bert.) O. Kuntze, Campos do Brasil Meridional e Mata Latifoliada da Encosta Atlântica (BERZAGHI, 1994), as quais estão organizadas em um mosaico estreitamente relacionado ao relevo, à drenagem $\mathrm{e}$ às formações superficiais e juntas compõem uma parte do bioma da Mata Atlântica (SÃO PAULO, 2006).

A formação Araucaria-Podocarpus de Campos do Jordão é composta por fragmentos florestais com fisionomias semelhantes, embora distintos em tamanho, forma e estado de conservação. No passado, essa região era utilizada somente para o cultivo e o pastoreio (MERLIM, 2005), mas, atualmente, divide espaço não só com as propriedades agrícolas, como também com as áreas urbanas, o que configura o atual grau de antropização dessa região, mesmo com os esforços para sua conservação.

Município está localizado na Serra da Mantiqueira que é o segundo maior conjunto de escarpas do Brasil Oriental, em uma região de relevo intensamente acidentado formada por altos espigões e fundos de vale com altitudes que variam entre 600 e $2000 \mathrm{~m}$. Essa região forma um planalto cristalino em bloco onde afloram terrenos constituídos por gnaisses, migmatitos, granitos, xistos, quartzitos, calcários, calciossilicáticas e anfibólitos, estando limitado por escarpas que se erguem, aproximadamente, $1500 \mathrm{~m}$ sobre as colinas do médio Vale do Paraíba (HASUI; OLIVEIRA, 1984).

O clima pode ser caracterizado, segundo Köppen (1948), como Cfb, subtropical de altitude, mesotérmico, úmido com verão temeprado. Os dados climáticos do município indicam uma precipitação média entre 1287,6 e $2128,0 \mathrm{~mm} \cdot \mathrm{ano}^{-1}$, com duas estações climáticas bem definidas, uma estação fria, pouco chuvosa, com temperatura média de $10^{\circ} \mathrm{C}$ e com cerca de $10 \mathrm{~mm}$ de precipitação, em julho, e outra mais úmida, em fevereiro, com temperatura média em torno de $18^{\circ} \mathrm{C}$ e precipitação acima de $300 \mathrm{~mm}$ (MERLIM, 2005). Nos meses de inverno, entre maio e agosto, ocorre redução dos índices pluviométricos, as geadas são freqüentes nesse período e as temperaturas podem variar entre $-0,2$ até $-4,9^{\circ} \mathrm{C}$ (SÃO PAULO, 2006).

O remanescente florestal estudado é um fragmento de formato triangular com 1,14ha (Figura 1) localizado próximo à latitude $22^{\circ} 41^{\prime} \mathrm{S}$ e longitude $45^{\circ} 26^{\circ} \mathrm{W}$, a 
$1882 \mathrm{~m}$ de altitude, razão pela qual é protegido como Área de Preservação Permanente pelo Código Florestal brasileiro (artigo 20, alínea "a", item " $h$ " da Lei 4.771/65 e suas revisões). Seu entorno imediato é composto principalmente por fragmentos florestais, além de campos antrópicos de pastagem, sítios de recreação e lazer e de estradas não pavimentadas que interligam loteamentos vizinhos e áreas residenciais.

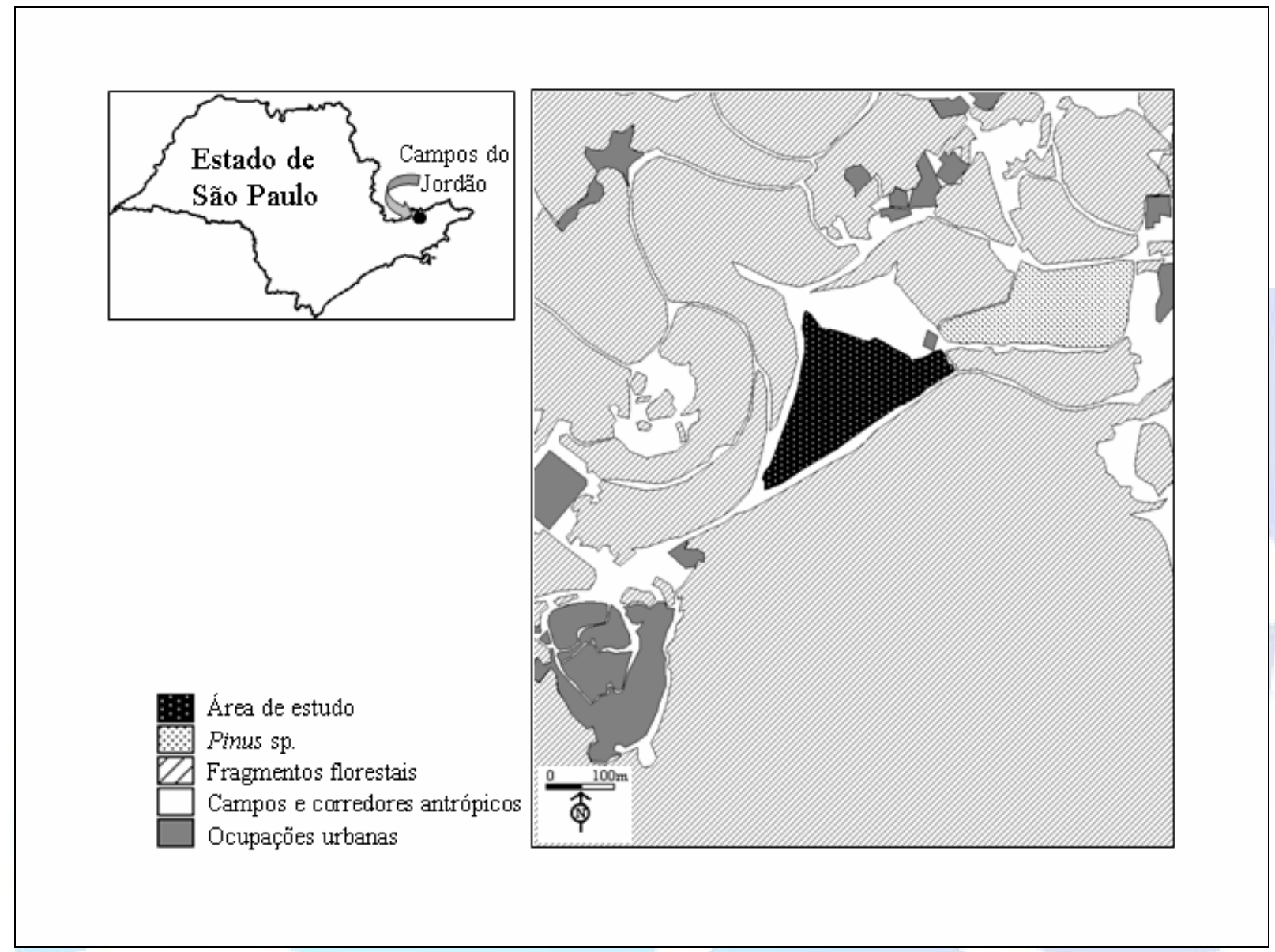

Figura 1. Croqui de localização do remanescente florestal estudado e sua posição em relação a outros fragmentos de Floresta Ombrófila Mista Alto Montana, plantio de Pinus sp. e ocupações urbanas de Campos do Jordão, SP. Áreas sem preenchimento correspondem aos campos e corredores (estradas vicinais) antrópicos.

O levantamento florístico da vegetação lenhosa foi realizado de setembro a dezembro de 2005 através do método de caminhamento de Figueiras et al. (1994), uma técnica de amostragem florística qualitativa expedita. A maioria das espécies foi registrada em campo, outras foram coletadas e posteriormente identificadas por comparação com as coleções de exsicatas do Herbário da Universidade Estadual de Campinas ou através de consultas à bibliografia especializada e a especialistas.

A lista de espécies foi organizada em famílias de acordo com o sistema proposto por Cronquist (1988). A nomenclatura das espécies foi organizada de acordo com a base de dados $\mathrm{W}^{3}$ Tropicos do Missouri Botanical Garden (MISSOURI 
BOTANICAL GARDEN, 2005) e de revisões taxonômicas recentes, adotando o sistema de autores proposto por Brumitt e Powell (1992).

Foram consideradas arbóreas as plantas com altura superior a três metros, com tronco bem definido e com ramificações acima de um metro do nível do solo e de hábito arbustivo, aquelas com até três metros de altura e com ramificação a partir de meio metro de altura do nível do solo (MEIRELLES, 2003).

As espécies observadas foram classificadas quanto ao seu hábitat em espécies de interior de floresta (In), de borda (Bd) e ocorrentes em ambos os hábitats (In/Bd). As espécies de interior de floresta foram classificadas também quanto à posição vertical na floresta (D - dossel, $\mathrm{Sb}$ - sub-bosque e $\mathrm{Cl}$ - clareira). Além disso, todas as espécies foram agrupadas em tipos de dispersão, zoocórica (zoo), anemocórica (ane) e autocórica (au) e em grupos sucessionais, pioneiras $(\mathrm{Pi})$, secundárias $(\mathrm{Sc})$ e nãocaracterizadas $(\mathrm{Nc})$, de acordo com os critérios de classificação sucessional proposto por Gandolfi (2000), modificado pelo agrupamento das secundárias iniciais e tardias em um único grupo.

\section{RESULTADOS E DISCUSSÃO}

Foram identificadas 54 espécies lenhosas, pertencentes a 43 gêneros e 33 famílias (Tabela 1). Prevaleceram espécies de hábito arbóreo (75\%) ocorrentes principalmente no sub-bosque do interior da floresta (38\%). No interior do fragmento florestal, a flora lenhosa revelou a existência de 35 espécies pertencentes a 31 gêneros e 26 famílias, enquanto que na área de borda foram identificadas apenas 10 espécies, pertencentes a cinco gêneros e seis famílias. Além dessas espécies exclusivas de interior ou de borda da floresta, foram identificadas outras nove espécies comuns a ambos os hábitats (Tabela 2).

$\mathrm{O}$ registro de espécies primitivas de refúgios alto-montanos, como Araucaria angustifolia, Podocarpus lambertii e Drymis brasiliensis (INSTITUTO BRASILEIRO DE GEOGRAFIA E ESTATÍSTICA, 1992) permite identificar o fragmento florestal estudado como Floresta Ombrófila Mista Alto Montana que, segundo (IBGE, 1992), corresponde a uma vegetação do tipo intertropical. Além dessas espécies, a ocorrência de Guatteria nigrescens, Vernonia diffusa, Cabralea canjerana, Roupala brasiliensis, Psychotria sessilis, Cupania vernalis, Clethra scabra, Ilex cerasifolia e Prunus myrtifolia (MEIRA-NETO et al. 1989, OLIVEIRAFILHO; FONTES, 2000, CARVALHO et al., 2005), reforça a evidência de ocorrência de uma fisionomia florestal Atlântica Alto-Montana.

O remanescente florestal da área urbana de Campos do Jordão possui apenas $15 \%$ do número total de espécies lenhosas observadas por Robim et al. (1990) na fitofisionomia florestal de araucária-podocarpo do Parque Estadual de Campos do Jordão (PECJ), o que pode demonstrar um comprometimento da sua fitofisionomia. Os resultados obtidos mostram que apenas $4,3 \%$ das espécies lenhosas e $25 \%$ das 
famílias registradas ocorrem em Floresta Semidecidual de Altitude estudadas por Meira-Neto et al. (1989), o que descarta a possibilidade do fragmento florestal estudado pertencer a esta fitofisionomia.

Apesar da intensa supressão ocorrida no decorrer da história, a Floresta Ombrófila Mista (FOM) continua sendo a vegetação dominante na paisagem da Mantiqueira. Trata-se de uma vegetação sujeita a sazonalidade do clima, o qual proporciona a manutenção de espécies típicas de florestas mistas que muitas vezes coexistem com espécies de outras florestas de altitude do Sul e Sudeste brasileiros (MEIRA-NETO et al., 1989).

Ainda que bem antropizado e empobrecido floristicamente em relação às outras áreas naturais com mesma fisionomia, o remanescente estudado possui diversos indivíduos de Araucaria angustifolia no dossel, no sub-bosque, na borda e no interior da floresta (Tabelas 1 e 2). Essa espécie é uma das únicas gimnospermas nativas brasileiras, tem comportamento pioneiro e produz sementes zoocóricas (LORENZI, 1992, BACKES; IRGANG, 2004, SÃO PAULO, 2006), além de estar presente na lista oficial de espécies da flora brasileira ameaçada de extinção na categoria Vulnerável (BRASIL, 1992), exigindo, portanto, a sua obrigatória conservação.

Drymis brasiliensis ocorreu no dossel e predominou no sub-bosque do interior da floresta (Tabela 1), trata-se de uma espécie pioneira cujos frutos são consumidos pela fauna (LORENZI, 1992, BACKES; IRGANG, 2004). Podocarpus lambertii é comum em áreas de vegetação secundária, tem comportamento pioneiro e, no interior da floresta, foi observada no dossel e no sub-bosque, predominando nesse segundo estrato como indivíduo de pequeno porte.

Outras espécies, como Myrsine umbellata e $M$. coriacea, foram observadas em grande quantidade na borda e no sub-bosque do interior da floresta (Tabela 1). Essa elevada ocorrência na área, aliadas à facilidade de germinação de suas sementes e ao comportamento agressivo em campos e capoeiras, evidencia que essas espécies desempenham importante papel na regeneração natural desse remanescente florestal.

O registro de adultos e observação de muitos indivíduos jovens de Roupala brasiliensis no sub-bosque e dossel do interior da floresta (Tabela 1), leva a crer que essa espécie está se estabelecendo com sucesso na área. As espécies Tibouchina mutabilis e Croton piptocalyx, ambas pioneiras, foram encontradas em abundância no dossel, no sub-bosque e na borda da floresta, indicando que o fragmento está passando por um processo sucessional.

Apenas 35\% das famílias encontradas no PECJ por Robim et al. (1990) foram registradas na área de estudo. Nessa Unidade de Conservação as famílias com maior número de espécies lenhosas foram, Asteraceae (15 espécies), Myrtaceae (12 espécies), Lauraceae (7 espécies) e Rubiaceae (7 espécies) (ROBIM et al., 1990), enquanto que para o remanescente do presente estudo, as famílias mais abundantes foram Asteraceae ( 8 espécies), Myrtaceae (6 espécies) e Melastomateceae (5 espécies). 
A vegetação não lenhosa mostrou grande diversidade de epífitas, representadas por bromélias e orquídeas e um abundante estrato herbáceo composto principalmente por gramíneas, carquejas, vassouras do gênero Baccharis sp. e macelas (Achyrocline satureoides (Lam.) DC). A abundância de epífitas, sobretudo bromélias, orquídeas, pteridófitas, musgos e líquens são características de florestas alto-montanas neotropicais (GENTRY, 1995) e as espécies de vassouras e carquejas são comuns em vegetação de Campo de Altitude (FERRI, 1980).

A classificação quanto ao tipo de dispersão mostrou que $31,4 \%$ das espécies dispersam suas sementes por zoocoria, $23,5 \%$ por anemocoria e 3,9\% por autocoria. Cerca de $41,2 \%$ das espécies não puderam ser caracterizadas por falta de informações na literatura. A maior proporção de espécies com síndrome zoocórica é comum em florestas tropicais (MORELLATO; LEITÃO FILHO, 1992, PENHALBER; MANTOVANI, 1997) onde a maior parte dos frutos é dispersa pelos animais e outra parte pelo vento, existindo poucas espécies autocóricas ou com outro tipo de dispersão (MORELLATO, 1995).

Os resultados obtidos mostram que espécies como Guatteria nigrescens, Ilex cerasifolia, Clethra scabra, Casearia sylvestris, Ocotea odorifera, Miconia albicans, Cabralea canjerana, Sorocea bomplandii, Myrcia fallax, Cupania vernalis, entre outras (Tabela 1), são zoocóricas e apresentam padrão contínuo de frutificação caracterizando um processo seqüencial durante todo o ano (MORELLATO; LEITÃO FILHO, 1992). Isso evidência a importância do fragmento como fonte de recursos para a fauna e como componente dispersor de propágulos no ambiente, característica importante para a regeneração e para a manutenção não só deste remanescente florestal, mas também de outros fragmentos florestais vizinhos.

A classificação das espécies em grupos sucessionais permitiu enquadrar $18 \%$ das espécies como pioneiras ( $\mathrm{Pi}$ ), $44 \%$ como secundárias $(\mathrm{Sc})$ e $38 \%$ como não caracterizadas $(\mathrm{Nc})$ devido à falta de informações na literatura. A classificação em grupos sucessionais tem sido muito polêmica em estudos de florestas tropicais em virtude da falta de conhecimentos sobre a autoecologia das espécies (RODRIGUES, 1995), mas representa um passo importante no entendimento da complexidade funcional dos sistemas naturais.

Uma grande parte das espécies pioneiras (6 espécies) e secundárias (19 espécies) foi encontrada no interior da floresta (Tabela 2) e esse elevado número de espécies Sc frente à pequena quantidade de $\mathrm{Pi}$ e a ocorrência de grande parte das espécies no sub-bosque (20 espécies) frente a um pequeno número delas em pequenas clareiras (4 espécies), indica a existência de um estágio intermediário de regeneração natural, em que, possivelmente, está ocorrendo um processo avançado de cicatrização de antigas clareiras.

A alteração da estrutura da paisagem é um processo que interfere na dinâmica das populações e altera os riscos de extinção e a probabilidade de deslocamento dessas populações (SOULÉ et al., 1992; ANDRÉN, 1994), resultando em mudanças na composição e na diversidade de comunidades (METZGER, 1999). A modificação 
na estrutura da paisagem do entorno imediato do fragmento em estudo pode ter sido um dos fatores que influenciaram na mudança da composição florística original do fragmento.

A ação humana tem criado ambientes propícios ao estabelecimento de espécies exóticas e ruderais agressivas nas bordas e no interior dos remanescentes de mata nativa (PRIMACK; RODRIGUES, 2001). Essa modificação iniciada pela fragmentação de áreas contínuas e conseqüente redução e isolamento dos remanescentes florestais de diversas formas e tamanhos (Figura 1), contribuiu para a alteração de proporção das áreas de hábitats (ANDRÉN, 1994), para o empobrecimento da flora lenhosa nativa e para a descaracterização da fitofisionomia original.

O tamanho reduzido do remanescente $(1,14 \mathrm{ha})$ possivelmente significou para muitas das espécies ora existentes, a ausência de uma área mínima necessária à sobrevivência (SAUNDERS et al., 1991 apud METZGER, 1999), causando o desaparecimento de espécies e o aumento da dominância de outras que conseguiram se manter no fragmento (HANSON et al., 1990). Concomitante, à redução de área os efeitos do isolamento e de borda podem ter influenciado negativamente a riqueza de espécies do interior da floresta.

Apesar do arranjo espacial dos fragmentos florestais da área de estudo não favorecer uma conectividade estrutural devido à inexistência de interseções por corredores naturais, o predomínio de espécies vegetais zoocóricas $(44,7 \%)$ evidencia uma conectividade funcional que pode influenciar indiretamente na abundância e na distribuição das plantas com sementes, especialmente aquelas disseminadas por vertebrados (VAN DORP; KALKHOVEN, 1988 apud METZGER, 1999).

$\mathrm{O}$ fragmento em estudo, assim como outros pequenos fragmentos remanescentes da paisagem (Figura 1) podem estar funcionando como "pontos de ligação" (stepping stones) que favorecerem a sobrevivência das metapopulações pela troca de fluxo gênico.

A fragmentação observada na área de estudo aumenta a vulnerabilidade dos remanescentes e favorece o desenvolvimento de espécies de borda e àquelas generalistas que ocorrem tanto na borda como no interior da floresta e que tendem a excluir as espécies de interior por competição ou por predação (METZGER, 1999, PRIMACK; RODRIGUES, 2001). O formato triangular do fragmento não favorece uma relação adequada de borda-área que minimize os efeitos de borda e de fragmentação, ao contrário, aproximam todos seus pontos da borda, especialmente na sua porção sul (Figura 1), facilitando o estabelecimento de espécies generalistas e agressivas no interior da floresta. Idealmente, áreas de forma circular minimizam essa relação e permitem que o centro do fragmento se mantenha mais distante da borda (PRIMACK; RODRIGUES, 2001).

A presença de espécies exóticas, como Platanus acerifolia no interior da floresta e Pinus ellioti e Cupressus sp. nas bordas (Tabela 1) provavelmente é resultado da inadequada relação borda-área do fragmento. São espécies utilizadas na 
arborização rural e urbana e em plantios na região de onde possivelmente foram dispersos para o fragmento. Além disso, foi verificada a presença de pequenas clareiras e de diversas trilhas que circundam e cruzam o fragmento, além de sinais de pastejo animal, corte de árvores e estradas vicinais no entorno da propriedade.

A ação humana na região possivelmente está criando condições ambientais que favorecem o estabelecimento dessas espécies exóticas nas bordas e no interior dos fragmentos. A falta de controle dessas espécies poderá favorecer o aumento de sua abundância e deslocar as espécies nativas através de competição por limitação de recursos (PRIMACK; RODRIGUES, 2001), alterando os hábitats e comprometendo a sustentabilidade das espécies nos fragmentos florestais. A fragmentação interna desse remanescente deve ser impedida a fim de se manter a integridade das populações naturais, de se evitar o surgimento de novas bordas e de minimizar outros efeitos negativos da fragmentação.

O remanescente de Floresta Ombrófila Mista Alto Montana estudado possui alterações antrópicas importantes que precisam ser atenuadas. O planejamento e a consolidação de estratégias de conservação, além da conscientização da população da região, podem ser os passos iniciais para a manutenção dessa área e de sua paisagem de entorno como uma reserva natural de diversidade em área urbana. Os trechos bem conservados dessa formação florestal de Campos do Jordão poderiam servir como base para estudos florísticos e planos de enriquecimento com espécies nativas dos remanescentes dessa fitofisionomia, permitindo uma reestruturação ambiental capaz de resistir aos impactos negativos causados na região.

\section{CONCLUSÕES}

Considerando os impactos negativos do processo de fragmentação atuante na região, o tamanho, o isolamento estrutural e o formato do fragmento, provavelmente, foram os fatores determinantes para o empobrecimento florístico e a descaracterização de sua fitofisionomia original. Apesar do baixo número de espécies registradas na área, esse fragmento pode ser de grande importância para a sustentabilidade dos remanescentes vizinhos se comprovado seu papel na conectividade funcional da paisagem. Além disso, nesse remanescente florestal, ainda podem ser encontradas espécies arbustivas e arbóreas comuns às formações florestais estacionais e mistas de altitude, que demonstram sua função de manutenção da ameaçada biodiversidade regional.

\section{AGRADECIMENTOS}

Os autores agradecem ao biólogo, Msc. em Biologia Vegetal, Leonardo Dias Meirelles pela identificação de algumas espécies apresentadas nesse trabalho. 


\section{REFERÊNCIAS}

ANDRÉN, H. Effects of habitat fragmentation on birds and mammals in landscapes with different proportions of suitable habitat: a review. Oikos, Lund, v. 71, n. 3, p. 355-366, 1994.

Disponível em: http://www.jstor.org/view/00301299/ap060188/06a00040/0 Acesso em: 31 jan. 2007.

BACKES, P.; IRGANG, B. Mata Attântica: as árvores e a paisagem. Porto Alegre: Paisagem do Sul Editora, 2004.

BERZAGHI, A. J. P. Dinâmica de serapilheira em mata de Auracaria e Podocarpus do Parque Estadual de Campos do Jordão, SP. 1994. $118 \mathrm{f}$. Dissertação (Mestrado em Ecologia) - Instituto de Biociências, Universidade de São Paulo, São Paulo, 1994.

BRASIL. Instituto Brasileiro do Meio Ambiente e dos Recursos Naturais Renováveis. Portaria IBAMA n ${ }^{\circ}$-N, de 15 de janeiro de 1992. Estabelece a lista oficial de espécies da flora brasileira ameaçada de extinção. Diário Oficial da União, Poder Executivo, Brasília, DF, 23 jan. 1992. Seção 1, v. 130, n. 16, p. 870-872. Disponível em: http://www.projetoselva.com.br/normas/portibama6n_92.doc Acesso em: 31 jan. 2007.

BRUMITT, R. K.; POWELL, C. E. Authors of plant names. Kew: Royal Botanic Gardens, 1992.

CARVALHO, D. A.; OLIVEIRA-FILHO, A. T.; VAN DEN BERG, E.; FONTES, M. A. L.; VILELA, E. A.; MARQUES, J. J. G. S. M.; CARVAlHO, W. A. C. Variações florísticas e estruturais do componente arbóreo de uma floresta ombrófila alto-montana às margens do Rio Grande, Bocaina de Minas, MG, Brasil = Structural and floristic variations of the arboreal component of a tropical upper montane rainforest on the margin of the Rio Grande, Bocaina de Minas, Brazil. Acta Botanica Brasílica, São Paulo, v. 19, n. 1, p. 91-109, jan./mar. 2005. http://dx.doi.org/10.1590/S0102-33062005000100010

CESTARO, L. A. A vegetação no ecossistema urbano. In: ENCONTRO NACIONAL SOBRE ARBORIZAÇÃO URBANA, 2., 1985, Porto Alegre. Anais... Porto Alegre: Secretaria Municipal de Meio Ambiente, 1985. p. 51-56.

CRONQUIST, A. The evolution and classification of flowering plants. New York: The New York Botanical Garden, 1988.

FERRI, M. G. Vegetação brasileira. Belo Horizonte: Editora Itatiaia, 1980. 
FIGUEIRAS, T. S.; NOGUEIRA, P. E.; BROCHADO, A. L.; GUALA, I. I. G. F. Caminhamento: um método expedito para levantamentos florístico qualitativo. Cadernos de Geociências, Rio de Janeiro, n. 12, p. 39-43, 1994.

FUNDAÇÃO SOS MATA ATLÂNTICA. Atlas da evolução dos remanescentes florestais e ecossistemas associados no domínio da Mata Attântica no período 1990-1995. São Paulo, 1998.

GANDOLFI, S. História natural de uma floresta estacional semidecidual no Município de Campinas (São Paulo, Brasil). 2000. 520 f. Tese (Doutorado em Biologia Vegetal) -Instituto de Biologia, Universidade Estadual de Campinas, Campinas, 2000.

GENTRY, A. H. Patterns of diversity and floristic composition in neotropical montane forests. In: CHURCHILL, S. P. (Org.). Biodiversity and conservation of neotropical montane forests: proceedings of neotropical montane forest biodiversity and conservation symposium. New York: The New York Botanical Garden, 1995. p. 103-126.

HANSON, J. S.; MALASON, G. P.; ARMSTRONG, M.P. Landscape fragmentation and dispersal in a model of riparian forest dynamics. Ecological Modelling, Amsterdam, v. 49, n. 3/4, p. 277-296, 1990.

HASUI, Y; OLIVEIRA, M. A. F. A Província Mantiqueira - Setor Central. In: ALMEIDA, F. F. M.; HASUI, Y. (Coord.). O pré-cambriano do Brasil. São Paulo: Edgard Blücher, 1984.

INSTITUTO BRASILEIRO DE GEOGRAFIA E ESTATÍSTICA. Manual técnico da vegetação brasileira. Rio de Janeiro, 1992.

KÖPPEN, W. Climatologia. Cidade do México: Editora Fondo de Cultura Econômica, 1948.

LORENZI, H. Árvores brasileiras: manual de identificação e cultivo de plantas arbóreas nativas do Brasil. Nova Odessa: Plantarum, 1992.

MEIRA-NETO, J. A. A.; BERNACCI, L. C.; GROMBONE, M. T.; TAMASHIRO, J. Y; LEITÃO FILHO, H. F. Composição florística da floresta semidecídua de altitude do Parque Municipal da Grota Funda (Atibaia, Estado de São Paulo). Acta Botanica Brasílica, São Paulo, v. 3, n. 2, p. 51-74, 1989. 
MEIRELLES, L. D. Florística das fisionomias vegetacionais e estrutura da floresta alto-montana de Monte Verde, Serra da Mantiqueira, MG. 2003. $150 \mathrm{f}$. Dissertação (Mestrado em Biologia Vegetal) - Instituto de Biologia, Universidade Estadual de Campinas, Campinas, 2003.

MERLIM, A. O. Macrofauna edáfica em ecossistemas preservados e degradados de araucária no Parque Estadual de Campos do Jordão, SP. 2005. 89 f. Dissertação (Mestrado em Ecologia de Agroecossistemas) - Escola Superior de Agricultura Luiz de Queiroz, Universidade de São Paulo, Piracicaba, 2005. Disponível em: http://www.teses.usp.br/teses/disponiveis/91/91131/tde-21062005144943/publico/ AnalyMerlim.pdf Acesso em 31 jan. 2007.

METZGER, J. P. Estrutura da paisagem e fragmentação: análise bibliográfica. Anais da Academia Brasileira de Ciências, Rio de Janeiro, v.71, n. 3-I, p. 445-463, 1999.

MISSOURI BOTANICAL GARDEN. W3 trópicos. Disponível em: http://mobot.org/W3T/Search/vast.html Acesso em: 2 ago. 2005.

MORELLATO, L. P. C.; LEITÃO FILHO, H. F. Padrões de frutificação e dispersão na Serra do Japi. In: MORELLATO, L. P. C. (Org.). História natural da Serra do Japi: ecologia e preservação de uma área florestal no sudeste do Brasil. Campinas: Editora da Unicamp/Fapesp, 1992. p. 112-140.

MORELlATO, L. P. C. As estações do ano na floresta. In: MORELLATO, P. C.; LEITÃO-FILHO, H. F. (Org.) Ecologia e preservação de uma floresta tropical urbana: reserva de Santa Genebra. Campinas: Editora da Unicamp, 1995. p. 37-41.

NORGAARD, R. O crescimento da economia global de trocas e a perda de diversidade biológica. In: WILSON, E. O. (Org.). Biodiversidade. Rio de Janeiro: Nova Fronteira, 1997. p. 261-268.

OLIVEIRA-FILHO, A. T.; FONTES, M. A. L. Patterns of floristic differentiation among Atlantic Forests in southeastern Brazil and the influence of climate. Biotropica, Los Angeles, v. 32, n. 4b, p. 793-810, 2000.

PENHALBER, E. F.; MANTOVANI, W. Floração e chuva de sementes em mata secundária em São Paulo, SP. Revista Brasileira de Botânica, São Paulo, v. 20, n. 2, p. 205-230, 1997.

PRIMACK, R. B.; RODRIGUES, E. Biologia da conservação. Londrina: Midiograf, 2001. 
ROBIM, M. J.; PASTORE, J. A.; AGUIAR, O. T.; BAITELLO, J. B. Flora arbóreoarbustiva e herbácea do Parque Estadual de Campo do Jordão (SP). Revista do Instituto Florestal, São Paulo, v. 2, n. 1, p. 31-53, 1990.

RODRIGUES, R. R. A sucessão florestal. In: MORELLATO, P. C.; LEITÃOFILHO, H. F. (Org.) Ecologia e preservação de uma floresta tropical urbana: reserva de Santa Genebra. Campinas: Editora da Unicamp, 1995. p. 30-36.

RUSCZYK, A. Análise da cobertura vegetal da cidade de Porto Alegre, RS. Revista Brasileira de Botânica, São Paulo, v. 9, p. 225-229, 1986.

SÃO PAULO (Estado). Secretaria do Meio Ambiente. Coordenadoria de Planejamento Ambiental Estratégico e Educação Ambiental. Mantiqueira: o castelo das águas. São Paulo, 2006.

SOUlÉ, M. E.; ALBERTS, A. C.; BOLGER, D. T. The effects of habitat fragmentation on chaparral plants and vertebrates. Oikos, Lund, v. 63, n. 1, p. 39-47, 1992.

WERNECK, M. S. A vegetação urbana espontânea de áreas baldias em um gradiente de urbanização, Belo Horizonte, MG. Bios: Cadernos do Departamento de Ciências Biológicas da PUC Minas, Belo Horizonte, v. 6, n. 6, p. 33-43, 1998. 


\begin{tabular}{|l|r|}
\hline Recebido em: 02/03/2006 & HOLOS Environment, v.7 n.2, 2007 - P. 168 \\
\hline Liberado para Publicação em: $10 / 11 / 2007$ & ISSN:1519-8634 (ON-LINE) \\
\hline
\end{tabular}

Tabela 1. Espécies arbustivas (arbs) e arbóreas (arbo) identificadas na área de interior da floresta (In) e na área de vegetação de borda (Bd) classificadas quanto ao seu estágio sucessional (pi - pioneira; sc - secundária e nc - não caracterizada), posição vertical quando dentro da floresta ( $\mathrm{D}$ - dossel; $\mathrm{Sb}$ - sub-bosque; $\mathrm{Cl}$ - clareira) e tipos de dispersão (zoocórica - zoo; anemocórica - ane; autocórica - au) Campos do Jordão, SP.

\begin{tabular}{|c|c|c|c|c|c|}
\hline \multirow{2}{*}{$\begin{array}{l}\text { Família/Espécie } \\
\text { ANNONACEAE }\end{array}$} & \multicolumn{5}{|c|}{ Hábito Hábitat Posição Dispersão Sucessão } \\
\hline & & & & & \\
\hline $\begin{array}{l}\text { Guatteria nigrescens Mart. } \\
\text { AOUIFOLIACEAE }\end{array}$ & arbo & In & $\mathrm{D}$ & zoo & $\mathrm{sc}$ \\
\hline $\begin{array}{l}\text { Ilex cerasifolia } \text { Reiss. } \\
\text { ARALIACEAE }\end{array}$ & arbo & In & $\mathrm{Sb}$ & $\mathrm{nc}$ & $\mathrm{sc}$ \\
\hline $\begin{array}{l}\text { Didymopanax calvus (Cham.) Decne. \& Planch. } \\
\text { ARAUCARIACEAE }\end{array}$ & arbo & In & $\mathrm{D}$ & zoo & $\mathrm{sc}$ \\
\hline $\begin{array}{l}\text { Araucaria angustifolia (Bertol.) Kuntze } \\
\text { ASTERACEAE }\end{array}$ & arbo & $\mathrm{Bd} / \mathrm{In}$ & $\mathrm{D} / \mathrm{Sb}$ & zoo & $\mathrm{sc}$ \\
\hline Baccharis sp.1 & arbs & $\mathrm{Bd}$ & & $\mathrm{nc}$ & $\mathrm{nc}$ \\
\hline Baccharis sp.2 & arbs & $\mathrm{Bd}$ & & $\mathrm{nc}$ & $\mathrm{nc}$ \\
\hline Baccharis sp. 3 & arbs & $\mathrm{Bd}$ & & $\mathrm{nc}$ & $\mathrm{nc}$ \\
\hline Baccharis sp.4 & arbs & $\mathrm{Bd}$ & & $\mathrm{nc}$ & $\mathrm{nc}$ \\
\hline Baccharis timera (Less.) DC. & arbs & $\mathrm{Bd}$ & & ane & nc \\
\hline Piptocarpha sp. & arbs & In & $\mathrm{D}$ & $\mathrm{nc}$ & $\mathrm{nc}$ \\
\hline Vernonia diffusa Less. & arbo & In & $\mathrm{Sb}$ & ane & pi \\
\hline Asteraceae sp.1 & arbs & $\mathrm{Bd} / \mathrm{In}$ & $\mathrm{Cl}$ & $\mathrm{nc}$ & $\mathrm{nc}$ \\
\hline Asteraceae sp. 2 & arbs & $\mathrm{Bd} / \mathrm{In}$ & $\mathrm{Cl}$ & $\mathrm{nc}$ & $\mathrm{nc}$ \\
\hline BIGNONIACEAE & & & & & \\
\hline $\begin{array}{l}\text { Jacaranda puberula Cham. } \\
\text { BOMBACACEAE }\end{array}$ & arbo & $\mathrm{Bd} / \mathrm{In}$ & $\mathrm{D}$ & ane & $\mathrm{sc}$ \\
\hline $\begin{array}{l}\text { Eriotheca candolleana (K. Schum.) A. Robyns } \\
\text { CLETHRACEAE }\end{array}$ & arbo & In & $\mathrm{Sb}$ & ane & $\mathrm{sc}$ \\
\hline $\begin{array}{l}\text { Clethra scabra Pers. } \\
\text { CUNONIACEAE }\end{array}$ & arbo & In & $\mathrm{Sb}$ & ane & $\mathrm{sc}$ \\
\hline $\begin{array}{l}\text { Weinmannia paulliniifolia } \text { Pohl } \\
\text { CUPRESSACEAE }\end{array}$ & arbo & In & $\mathrm{D}$ & ane & sc \\
\hline $\begin{array}{l}\text { Cupressus sp. } \\
\text { CYATHEACEAE }\end{array}$ & arbo & $\mathrm{Bd}$ & & - & ex \\
\hline $\begin{array}{l}\text { Alsophila sp. } \\
\text { ERICACEAE }\end{array}$ & arbo & In & $\mathrm{Sb}$ & ane & nc \\
\hline $\begin{array}{l}\text { Gaultheria } s p . \\
\text { EUPHORBIACEAE }\end{array}$ & arbs & $\mathrm{Bd}$ & - & $\mathrm{nc}$ & $\mathrm{nc}$ \\
\hline Alchornea glandulosa Poepp. & arbo & In & $\mathrm{D} / \mathrm{Sb}$ & $\mathrm{au}$ & pi \\
\hline $\begin{array}{l}\text { Croton piptocalyx Müll. Arg. } \\
\text { FLACOURTIACEAE }\end{array}$ & arbo & $\mathrm{Bd} / \mathrm{In}$ & $\mathrm{D} / \mathrm{Sb}$ & au & pi \\
\hline $\begin{array}{l}\text { Casearia sylvestris Sw. } \\
\text { LAURACEAE }\end{array}$ & arbo & In & $\mathrm{D}$ & zoo & pi \\
\hline Ocotea odorifera (Vellozo) Rohwer & arbo & In & $\mathrm{Sb}$ & zoo & $\mathrm{sc}$ \\
\hline Ocotea pulchella (Nees) Mez & arbo & In & $\mathrm{Sb}$ & zoo & $\mathrm{sc}$ \\
\hline
\end{tabular}

Continuação 


\begin{tabular}{|l|r|}
\hline Recebido em: 02/03/2006 & HOLOS Environment, v.7 n.2, 2007 - P. 169 \\
\hline Liberado para Publicação em: $10 / 11 / 2007$ & ISSN:1519-8634 (ON-LINE) \\
\hline
\end{tabular}

\begin{tabular}{|c|c|c|c|c|c|}
\hline \multirow{2}{*}{$\begin{array}{l}\text { Família/Espécie } \\
\text { MALPIGHIACEAE }\end{array}$} & \multicolumn{5}{|c|}{ Hábito Hábitat Posição Dispersão Sucessão } \\
\hline & & & & & \\
\hline Byrsonima sp. & arbs & $\mathrm{Bd}$ & - & $\mathrm{nc}$ & nc \\
\hline MELASTOMATACEAE & & & & & \\
\hline Leandra carassana (DC.) Cogn. & arbs & $\mathrm{Bd} / \mathrm{In}$ & $\mathrm{Cl}$ & $\mathrm{nc}$ & $\mathrm{nc}$ \\
\hline Miconia albicans (Sw.) Triana & arbs & In & $\mathrm{Cl}$ & zoo & pi \\
\hline Miconia langsdorffii Cogn. & arbs & In & $\mathrm{Sb}$ & nc & nc \\
\hline Tibouchina granulosa (Desr.) Cogn. & arbo & In & $\mathrm{Sb}$ & ane & pi \\
\hline $\begin{array}{l}\text { Tibouchina mutabilis (Vell.) Cogn. } \\
\text { MELIACEAE }\end{array}$ & arbo & $\mathrm{Bd} / \mathrm{In}$ & $\mathrm{D} / \mathrm{Sb}$ & ane & pi \\
\hline $\begin{array}{l}\text { Cabralea canjerana (Vell.) Mart. } \\
\text { MIMOSACEAE }\end{array}$ & arbo & In & $\mathrm{D}$ & zoo & sc \\
\hline Anadenanthera colubrina (Vell.) Brenan & arbo & In & $\mathrm{Sb}$ & ane & $\mathrm{sc}$ \\
\hline $\begin{array}{l}\text { Mimosaceae sp. } \\
\text { MONIMIACEAE }\end{array}$ & arbs & $\mathrm{Bd}$ & - & $\mathrm{nc}$ & $\mathrm{nc}$ \\
\hline $\begin{array}{l}\text { Macropeplus ligustrinus (Tul.) Perkins } \\
\text { MORACEAE }\end{array}$ & arbo & In & $\mathrm{Sb}$ & nc & $\mathrm{nc}$ \\
\hline $\begin{array}{l}\text { Sorocea bonplandii (Baill.) W.C. Burger, Lanj. \& Wess. Boer } \\
\text { MYRSINACEAE }\end{array}$ & arbo & In & $\mathrm{Sb}$ & zoo & sc \\
\hline Myrsine coriacea (Sw.) R. Br. ex Roem. \& Schult. & arbo & $\mathrm{Bd} / \mathrm{In}$ & $\mathrm{Sb}$ & $\mathrm{nc}$ & pi \\
\hline $\begin{array}{l}\text { Myrsine umbellata Mart. } \\
\text { MYRTACEAE }\end{array}$ & arbo & $\mathrm{Bd} / \mathrm{In}$ & $\mathrm{Sb}$ & nc & $\mathrm{sc}$ \\
\hline Calyptranthes clusiifolia (Miq.) O. Berg & arbo & In & $\mathrm{Sb}$ & zoo & $\mathrm{nc}$ \\
\hline Myrcia brasiliensis Kiaersk. & arbo & In & $\mathrm{D}$ & $\mathrm{nc}$ & $\mathrm{nc}$ \\
\hline Myrcia fallax (Rich.) DC. & arbo & In & $\mathrm{Sb}$ & zoo & sc \\
\hline Myrcia guianensis (Aubl.) DC. & arbo & In & $\mathrm{D} / \mathrm{Sb}$ & nc & nc \\
\hline Pimenta pseudocaryophyllus (Gomes) Landrum & arbo & In & $\mathrm{D}$ & zoo & sc \\
\hline $\begin{array}{l}\text { Siphoneugena sp. } \\
\text { PINACEAE }\end{array}$ & arbo & In & $\mathrm{D}$ & $\mathrm{nc}$ & nc \\
\hline $\begin{array}{l}\text { Pinus elliottii Engelm. } \\
\text { PLATANACEAE }\end{array}$ & arbo & $\mathrm{Bd}$ & & - & ex \\
\hline $\begin{array}{l}\text { Platanus acerifolia (Aiton) Willd. } \\
\text { PODOCARPACEAE }\end{array}$ & arbo & In & $\mathrm{Sb}$ & - & ex \\
\hline $\begin{array}{l}\text { Podocarpus lambertii Klotzsch ex Endl. } \\
\text { PROTEACEAE }\end{array}$ & arbo & In & $\mathrm{D} / \mathrm{Sb}$ & ane & pi \\
\hline $\begin{array}{l}\text { Roupala brasiliensis Klotzsch } \\
\text { ROSACEAE }\end{array}$ & arbo & In & $\mathrm{D} / \mathrm{Sb}$ & ane & sc \\
\hline $\begin{array}{l}\text { Prunus myrtifolia (L.) Urb. } \\
\text { RUBIACEAE }\end{array}$ & arbo & In & $\mathrm{D}$ & zoo & sc \\
\hline $\begin{array}{l}\text { Psychotria sessilis Vell. } \\
\text { SAPINDACEAE }\end{array}$ & arbo & In & $\mathrm{Sb}$ & $\mathrm{nc}$ & nc \\
\hline $\begin{array}{l}\text { Cupania vernalis Cambess. } \\
\text { THEACEAE }\end{array}$ & arbo & In & $\mathrm{Sb}$ & zoo & sc \\
\hline $\begin{array}{l}\text { Gordonia fruticosa (Schrad.) H. Keng } \\
\text { TILIACEAE }\end{array}$ & arbo & In & $\mathrm{Sb}$ & zoo & $\mathrm{sc}$ \\
\hline $\begin{array}{l}\text { Luehea paniculata Mart. } \\
\text { WINTERACEAE }\end{array}$ & arbo & In & $\mathrm{D}$ & $\mathrm{nc}$ & sc \\
\hline Drimys brasiliensis Miers & arbo & In & $\mathrm{D} / \mathrm{Sb}$ & zoo & $\mathrm{sc}$ \\
\hline
\end{tabular}


Tabela 2. Número de espécies, de gêneros e de famílias, seus hábitos (Arbo arbóreo, Arbs - arbustivo), posição na floresta (D - dossel, $\mathrm{Sb}$ - sub-bosque, D/Sb ambos os estratos e $\mathrm{Cl}$ - clareira), categoria sucessional ( $\mathrm{Pi}$ - pioneira, $\mathrm{Sc}-$ secundária e $\mathrm{Nc}$ - não-caracterizadas) e espécies exóticas (Ex) para o remanescente de Floresta Ombrófila Mista Alto Montana, Campos do Jordão, SP.

\begin{tabular}{|c|c|c|c|c|c|c|c|c|c|c|c|c|c|}
\hline & \multicolumn{3}{|c|}{ Espécie Gênero Famíli } & \multicolumn{2}{|c|}{ Hábito } & \multicolumn{4}{|c|}{ Posição } & \multicolumn{3}{|c|}{ Sucessão } & \multirow[t]{2}{*}{ Ex } \\
\hline & & & & $\overline{\text { Arbo }}$ & $\overline{\mathrm{Arbs}}$ & $\overline{\mathrm{D}}$ & $\mathrm{Sb}$ & $\mathrm{D} / \mathrm{Sb}$ & $\mathrm{Cl}$ & $\overline{\mathrm{Pi}}$ & $\mathrm{Sc}$ & $\overline{\mathrm{Nc}}$ & \\
\hline$\overline{\text { Total }}$ & 54 & 43 & 33 & 40 & 14 & & & & & 9 & 22 & 20 & 3 \\
\hline Borda & 10 & 5 & 6 & 2 & 8 & & & & & 0 & 0 & 8 & 2 \\
\hline Interior & 35 & 31 & 26 & 32 & 3 & 10 & 0 & 0 & 4 & 6 & 19 & 9 & 1 \\
\hline Borda/Interior & 9 & 7 & 6 & 6 & 3 & 12 & 20 & 8 & 4 & 3 & 3 & 3 & 0 \\
\hline
\end{tabular}

